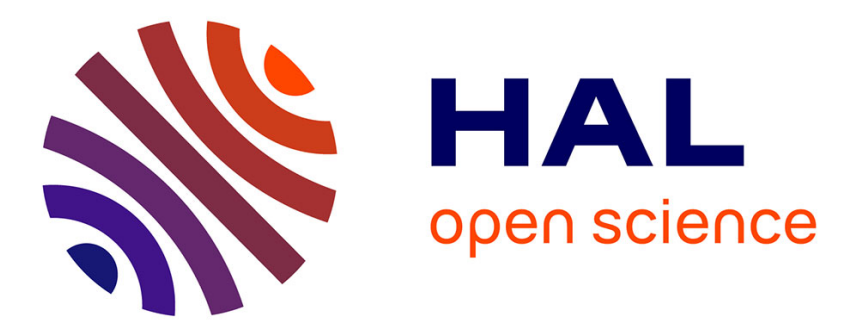

\title{
The Superconformal Gaugings in Three Dimensions
}

Eric Bergshoeff, Olaf Hohm, Diederik Roest, Henning Samtleben, Ergin Sezgin

\section{To cite this version:}

Eric Bergshoeff, Olaf Hohm, Diederik Roest, Henning Samtleben, Ergin Sezgin. The Superconformal Gaugings in Three Dimensions. Journal of High Energy Physics, 2008, 0809, pp.101. 10.1088/11266708/2008/09/101 . ensl-00299637

\section{HAL Id: ensl-00299637 https://hal-ens-lyon.archives-ouvertes.fr/ensl-00299637}

Submitted on 17 Jul 2008

HAL is a multi-disciplinary open access archive for the deposit and dissemination of scientific research documents, whether they are published or not. The documents may come from teaching and research institutions in France or abroad, or from public or private research centers.
L'archive ouverte pluridisciplinaire HAL, est destinée au dépôt et à la diffusion de documents scientifiques de niveau recherche, publiés ou non, émanant des établissements d'enseignement et de recherche français ou étrangers, des laboratoires publics ou privés. 
UG-08-11

UB-ECM-PF-08/16

MIFP-08-18

\title{
The Superconformal Gaugings in Three Dimensions
}

\author{
Eric A. Bergshoeff ${ }^{\dagger}$, Olaf Hohm ${ }^{\dagger}$, Diederik Roest ${ }^{*}$, \\ Henning Samtleben ${ }^{* *}$ and Ergin Sezgin ${ }^{\ddagger 1}$ \\ † Centre for Theoretical Physics, University of Groningen, \\ Nijenborgh 4, 9747 AG Groningen, The Netherlands \\ * Departament Estructura i Constituents de la Materia \\ $\&$ Institut de Ciències del Cosmos, \\ Universitat de Barcelona \\ Diagonal 647, 08028 Barcelona, Spain \\ ** Universite de Lyon, Laboratoire de Physique, \\ Ecole Normale Superieure de Lyon, \\ 46 allee dItalie, F-69364 Lyon Cedex 07, France \\ $¥$ George P. and Cynthia W. Mitchell Institute for Fundamental Physics, \\ Texas AEBM University, College Station, TX 77843-4242, U.S.A
}

\begin{abstract}
We show how three-dimensional superconformal theories for any number $\mathscr{N} \leq 8$ of supersymmetries can be obtained by taking a conformal limit of the corresponding three-dimensional gauged supergravity models. The superconformal theories are characterized by an embedding tensor that satisfies a linear and quadratic constraint. We analyze these constraints and give the general solutions for all cases. We find new $\mathscr{N}=4,5$ superconformal theories based on the exceptional Lie superalgebras $F(4), G(3)$ and $D(2 \mid 1 ; \alpha)$. Using the supergravity connection we discuss which massive deformations to expect. As an example we work out the details for the case of $\mathscr{N}=6$ supersymmetry.
\end{abstract}

July 2008

\footnotetext{
${ }^{1}$ e-mail: e.a.bergshoeff@rug.nl, o.hohm@rug.nl, droest@ecm.ub.es, henning.samtleben@ens-lyon.fr, sezgin@physics.tamu.edu
} 


\section{Introduction}

Three-dimensional superconformal theories have been studied intensively recently in view of their relevance to describing the dynamics of multiple M2-branes [1]. The starting point of this development was the construction of the $\mathscr{N}=8$ supersymmetric models by Bagger and Lambert [2-4] and Gustavsson [5,6]. These models are invariant under the symmetries of the $\mathrm{OSp}(8 \mid 4)$ superconformal algebra [7]. However, under certain assumptions, there is only a single $\mathscr{N}=8$ model with $\mathrm{SO}(4)$ gauging $[8,9]$.

A way to obtain more general gauge groups is to consider models with less superconformal symmetry, like $\mathscr{N}=1,2[10-13], \mathscr{N}=3[14,15]$ or $\mathscr{N}=4$ [16-18]. A particularly interesting class of models are the $\mathscr{N}=6$ models with $\mathrm{SU}(N) \times \mathrm{SU}(N)$ gauge groups [19]. Recently, three papers have appeared that deal with the general construction of $\mathscr{N}=6$ superconformal theories. First of all, in $[20] \mathscr{N}=6$ superconformal models were constructed starting from $\mathscr{N}=4$ supersymmetry with special matter multiplets and making use of a relation with Lie superalgebras [16]. Secondly, in [21] a general framework for constructing $\mathscr{N}=6$ superconformal gauge theories using the three-algebra approach was presented. Finally, a group-theoretical classification of the gauge groups and matter content of $\mathscr{N}=6$ superconformal gauge theories was given in [22].

In this note we wish to approach the construction of three-dimensional superconformal gauge theories for all values of $\mathscr{N}$ by making use of a relation with gauged supergravity $[23,24]$. Three-dimensional gauged supergravities have been constructed using the so-called embedding tensor technique. This method was originally developed in the construction of maximal $\mathscr{N}=16$ supergravities [25,26]. Here the most general $\mathscr{N}=16$ gaugings were classified, which are encoded in the "embedding tensor". The role of this tensor is to specify which subgroup of the global symmetry group is gauged and which vectors are needed to perform this gauging. Later, the same technique was applied to construct the matter-coupled half-maximal $\mathscr{N}=8$ theory $[27,28]$ as well as the $\mathscr{N}<8$ theories [28].

In [24] it was shown how the $\mathscr{N}=8$ model of [2-6] can be obtained by taking an appropriate limit to global supersymmetry of the $\mathscr{N}=8$ supergravity model of $[27,28]$. We will refer to this limit as the conformal limit. We consider here the conformal limit of all the other gauged supergravity models. In general, the embedding tensor characterizing the superconformal theory satisfies a set of linear and quadratic constraints. We show how these constraints can be determined from gauged supergravity by taking the conformal limit and find agreement with all known structures. Furthermore, we present a systematic way to solve these constraints, which reproduces the classification of superconformal theories for different values of $\mathscr{N}$ given in the recent literature $[16,18,20,22]$. Furthermore, inspired by a connection between superconformal gauge theories and Lie superalgebras observed in [16], we construct new $\mathscr{N}=4,5$ superconformal theories that are based on the exceptional Lie superalgebras $F(4), G(3)$ and $D(2 \mid 1 ; \alpha)$ with $\alpha$ a free parameter. They lead to superconformal theories with $\mathrm{SO}(7) \times \mathrm{Sp}(1), G_{2} \times \mathrm{Sp}(1)$ and $\mathrm{SO}(4) \times \mathrm{Sp}(1)$ gaugings, respectively ${ }^{2}$.

One advantage of the supergravity approach is that the same idea can be used to obtain non-conformal theories as well by taking other limits. A particularly interesting

\footnotetext{
${ }^{2}$ We thank J. Park for a stimulating discussion regarding the case of $F(4)$.
} 
class of models is obtained by taking the limit of a gauged supergravity where the gauge group lies entirely within the $R$-symmetry group. As was shown in [24] for the $\mathscr{N}=8$ case, such gaugings do not survive the limit to global conformal supersymmetry but nevertheless give rise to their massive deformation. Such massive deformations are important to test the idea of multiple M-branes and have been considered for $\mathscr{N}=8[29,30]$ and $\mathscr{N}=6[20,31]$.

This work is organized as follows. In section 2 we discuss the conformal limit and show how the properties of the embedding tensor characterizing superconformal theories in three dimensions can be derived from gauged supergravity. In particular, we derive the linear and quadratic constraints these tensors must satisfy for different values of $\mathscr{N}$. In section 3 we perform a systematic analysis of these constraints and derive the possible gauge groups and matter content. Next, we work out the details of our method for the specific example of $\mathscr{N}=6$ supersymmetry in section 4 . Finally, in section 5 we present our conclusions. In particular, we comment on the possible massive deformations. Appendix A explains our notation and conventions.

\section{Superconformal gaugings in three dimensions}

\subsection{Gauged supergravity}

We begin with a review of the possible gauged supergravity theories in three dimensions with different numbers $\mathscr{N}$ of supersymmetries. We are interested in theories with $\mathscr{N} \leq 8$ as these have matter multiplets (in addition to the supergravity multiplet) and allow for a limit to a globally supersymmetric field theory.

Three-dimensional supergravity theories differ from their higher-dimensional relatives in that all bosonic degrees of freedom can be described by scalar fields. These can be seen as coordinates of a manifold, on which supersymmetry imposes a number of geometric conditions [32]. For $\mathscr{N}>4$ these are strong enough to completely fix the (ungauged) theory: the scalar manifolds are given by certain symmetric spaces of the form

$$
\mathscr{M}=\frac{\hat{G}}{\hat{H}},
$$

where $\hat{G}$ is a simple Lie group of isometries, and $\hat{H}$ is its maximal compact subgroup. For lower values of $\mathscr{N}$, the scalar manifolds can be more general manifolds such as quaternionic, Kähler and Riemannian manifolds. However, for our purposes it will be sufficient to consider certain symmetric spaces for $\mathscr{N} \leq 4$ as well. The different cases are summarised in table 1 . Note that the $\mathscr{N}=4$ scalar manifold consists of a product of two quaternionic spaces. This possibility occurs due to the existence of two inequivalent $\mathscr{N}=4$ matter multiplets, hyper and twisted hyper multiplets. For other values of $\mathscr{N}$ there is a unique matter multiplet.

Turning to gauged supergravity, it is important to note that a special $D=3$ feature is that the gauge vectors have no independent kinetic term but only occur via a ChernSimons term. In this way they do not introduce new degrees of freedom but are dual to the scalar fields. More precisely, one can introduce as many vector fields as there are isometries on the scalar target space. 


\begin{tabular}{||c||c|c||c||}
\hline $\mathscr{N}$ & $\hat{G}$ & $\hat{H}$ & $\operatorname{dim}[\hat{G} / \hat{H}]$ \\
\hline \hline 8 & $\mathrm{SO}(8, N)$ & $\mathrm{SO}(8) \times \mathrm{SO}(N)$ & $8 N$ \\
\hline 6 & $\mathrm{SU}(4, N)$ & $\mathrm{S}(\mathrm{U}(4) \times \mathrm{U}(N))$ & $8 N$ \\
\hline 5 & $\mathrm{Sp}(2, N)$ & $\mathrm{Sp}(2) \times \mathrm{Sp}(N)$ & $8 N$ \\
\hline 4 & $\mathrm{Sp}(1, N) \times \mathrm{Sp}\left(1, N^{\prime}\right)$ & $\mathrm{Sp}(1) \times \mathrm{Sp}(N) \times \mathrm{Sp}(1) \times \mathrm{Sp}\left(N^{\prime}\right)$ & $4 N+4 N^{\prime}$ \\
\hline 3 & $\mathrm{Sp}(1, \mathrm{~N})$ & $\mathrm{Sp}(1) \times \mathrm{Sp}(N)$ & $4 N$ \\
\hline 2 & $\mathrm{SU}(1, N)$ & $\mathrm{S}(\mathrm{U}(1) \times \mathrm{U}(N))$ & $2 N$ \\
\hline 1 & $\mathrm{SO}(1, N)$ & $\mathrm{SO}(N)$ & $N$ \\
\hline
\end{tabular}

Table 1: The isometry and isotropy groups $\hat{G}$ and $\hat{H}$ of the symmetric scalar manifolds of three-dimensional $\mathscr{N}$-extended supergravity and their dimensions. For $N \leq 4$ we have just included a particular series of symmetric spaces, as it turns out that these contain the most general global limit to flat target space..

The possible gaugings of $D=3$ supergravity theories have been classified using the embedding tensor approach $[25,26,28]$. The embedding tensor $\Theta_{\alpha \beta}=\Theta_{\beta \alpha}$ takes values in the symmetric product of the adjoint representation of the global symmetry group $\hat{G}$ :

$$
\Theta \in(\operatorname{Adj}(\hat{G}) \otimes \operatorname{Adj}(\hat{G}))_{\text {symm }},
$$

and relates gauge vectors to generators of $\hat{G}$. The associated transformations are then gauged due to the introduction of the embedding tensor in covariant derivatives which take the general form

$$
D_{\mu}=\partial_{\mu}-A_{\mu}^{\alpha} \Theta_{\alpha \beta} t^{\beta},
$$

for some representation matrices $t^{\beta}$ of $\hat{G}$. Note that $X_{\alpha}=\Theta_{\alpha \beta} t^{\beta}$ denote the generators whose symmetries are being gauged. Also, the embedding tensor appears as a metric in the Chern-Simons term

$$
\mathscr{L}_{\mathrm{CS}}=-\frac{1}{2} \varepsilon^{\mu \nu \rho} A_{\mu}{ }^{\alpha} \Theta_{\alpha \beta}\left(\partial_{\nu} A_{\rho}{ }^{\beta}-\frac{1}{3} \Theta_{\gamma \delta} f^{\beta \delta}{ }_{\epsilon} A_{\nu}{ }^{\gamma} A_{\rho}{ }^{\epsilon}\right)
$$

with vector fields transforming in the adjoint of $\hat{G}$ and the structure constants $f^{\alpha \beta}$ of the global symmetry group $\hat{G}$. In supergravity there is a number of restrictions on which transformations can be gauged. These can be succinctly summarised in terms of a linear and a quadratic constraint on the embedding tensor.

The quadratic constraint follows from the requirement that the embedding tensor itself is invariant under the transformations that are gauged due to the introduction of $\Theta$. This condition takes the same form for all values of $\mathscr{N}$ :

$$
\Theta_{\alpha \beta} \Theta_{\gamma(\delta} f_{\epsilon)}^{\alpha \gamma}=0 .
$$

In case the embedding tensor projects onto a semisimple subgroup of $\hat{G}$ and is expressed in terms of invariant tensors of that subgroup, the quadratic constraint (2.5) is automatically satisfied. 
The linear constraint on the embedding tensor follows from supersymmetry. In other words, it is perfectly consistent to introduce gaugings that do not satisfy the linear constraint, but these will not preserve supersymmetry. As it follows from the requirement of supersymmetry, this condition takes a different form for different values of $\mathscr{N}$ :

- $\mathscr{N}=8$ supergravity:

The embedding tensor takes values in the symmetric product of the adjoint of $\hat{G}=$ $\mathrm{SO}(8, N)$. Representing the adjoint index $\alpha$ by a pair of antisymmetric fundamental indices, i.e. $\alpha=[A B]$, the embedding tensor is of the form $\Theta_{[A B],[C D]}$ and in terms of $\mathrm{SO}(8, N)$ Young tableaux ${ }^{3}$ decomposes according to

$$
(\boxminus \otimes \boxminus)_{\mathrm{symm}}=1 \oplus \square \oplus \boxminus \oplus \boxminus .
$$

Supersymmetry requires absence of the last representation corresponding to the window tableau.

- $\mathscr{N}=6$ supergravity:

The embedding tensor takes values in the symmetric product of the adjoint of $\hat{G}=$ $\mathrm{SU}(4, N)$. Representing the adjoint index $\alpha$ by a lower fundamental index $A$ and an upper anti-fundamental index $B$, i.e. $\alpha=\left({ }_{A}{ }^{B}\right)$, the embedding tensor is of the form $\Theta_{A}{ }^{B}, C_{C}{ }^{D}$ and in terms of $\mathrm{SU}(4, N)$ Young tableaux decomposes according to ${ }^{4}$ :

$$
(\square \bar{\square} \otimes \square \bar{\square})_{\mathrm{symm}}=1 \oplus \square \bar{\square} \oplus \boxminus \overline{\bar{G}} \oplus \square \bar{\square} .
$$

As supersymmetry requires the latter representation to vanish, the embedding tensor is anti-symmetric in its lower two indices: $\left.\Theta_{A}{ }^{B}, C^{D}=\Theta_{[A}^{B}, C\right]^{D}$.

- $\mathscr{N}=5$ supergravity:

The embedding tensor takes values in the symmetric product of the adjoint of $\hat{G}=$ $\operatorname{Sp}(2, N)$. Representing the adjoint index $\alpha$ by a pair of symmetric fundamental indices, i.e. $\alpha=(A B)$, the embedding tensor is of the form $\Theta_{(A B),(C D)}$ and in terms of $\operatorname{Sp}(2, N)$ Young tableaux decomposes according to

$$
(\square \otimes \square)_{\mathrm{symm}}=1 \oplus \boxminus \oplus \boxminus \oplus \square \square .
$$

The latter representation again has to be absent for supersymmetric gaugings, leading to the following constraint on the embedding tensor: $\Theta_{(A B, C D)}=0$.

- $\mathscr{N}=4$ supergravity:

In this case the embedding tensor consists of three parts. One part, $\Theta_{A B, C D}$, takes values in the symmetric product of the adjoint of $\operatorname{Sp}(1, N)$ and satisfies the analogous conditions as for $\mathscr{N}=5$. Similarly, the other part $\Theta_{A^{\prime} B^{\prime}, C^{\prime} D^{\prime}}$ takes values in the symmetric product

\footnotetext{
${ }^{3}$ Here, we use Young tableaux of $\mathrm{SO}(8, \mathrm{~N})$ in which symmetrization refers to traceless symmetrization, such that the representations are irreducible.

${ }^{4}$ We use here a notation where a (barred) Young tableau denotes (upper) lower indices of a tensor and traces are subtracted.
} 
of the adjoint of $\operatorname{Sp}\left(1, N^{\prime}\right)$ and is subject to the same conditions. The last part, $\Theta_{A B, C^{\prime} D^{\prime}}$ takes values in the product of the adjoints of both factors of the global symmetry group $\hat{G}$ and is not subjected to any linear constraint.However, it does have to satisfy a quadratic condition:

$$
\Theta_{\alpha \beta} \Theta_{\gamma \epsilon^{\prime}} f_{\delta}^{\alpha \gamma}+\Theta_{\alpha^{\prime} \beta} \Theta_{\gamma^{\prime} \delta} f_{\epsilon^{\prime}}^{\alpha^{\prime} \gamma^{\prime}}=0
$$

and similar for $\left(\alpha \leftrightarrow \alpha^{\prime}\right)$.

- $\mathscr{N}<3$ supergravity:

In these cases supersymmetry does not impose any linear condition: all consistent gaugings (satisfying the quadratic constraint) are compatible with supersymmetry. In addition, for $\mathscr{N}=1,2$, there are deformations that do not correspond to any gauging but to the introduction of a superpotential instead [28].

There are in general two strategies to solve the set of linear and quadratic constraints on the embedding tensor. Either one starts from an embedding tensor which projects onto a given subgroup by means of an invariant tensor such that the quadratic constraint is automatically satisfied. In this case, the linear constraint becomes a non-trivial identity which decides if the gauging is a viable one. Alternatively, one may start from the general solution of the linear constraint which can directly be expressed in terms of the proper subrepresentations. Then, the quadratic constraint becomes a non-trivial identity which selects the proper gaugings. In both cases the embedding tensor represents the CartanKilling metric of the gauge group. However, while in solving the quadratic constraint first, we can specify the Cartan-Killing metric in any basis we like, e.g. the diagonal one. If we solve the linear constraint first instead, the subsequent solution of the quadratic constraint, if it exists at all, yields the Cartan-Killing metric in a particular basis over which we no longer have control, e.g. it may emerge in a non-diagonal basis.

\subsection{The conformal limit}

We now turn to the conformal limit, whose aim is to extract superconformal theories from the gauged supergravities discussed above. This limit was performed explicitly for $\mathscr{N}=8$ in [24] and will be generalised here to lower values of $\mathscr{N}$.

It will be instructive to first discuss the limit to global supersymmetry in the ungauged case. Upon sending Newton's constant to zero, the supergravity and matter multiplets decouple, and the former will be set to zero. The resulting theory for the matter multiplets has $\mathscr{N}$ global supersymmetries. The isometry groups of supergravity, see table 1, can be seen to split up into three parts. Its compact part, which is the product of the Rsymmetry group $H_{\mathrm{R}}=\mathrm{SO}(\mathscr{N})$ and its orthogonal complement $G$, are unaffected by the global limit. In contrast, the non-compact generators reduce to nilpotent generators that transform under the compact parts:

$$
\hat{G} \rightarrow\left(G \times H_{\mathrm{R}}\right) \ltimes \mathbb{R}^{c N},
$$

for integer $N$ and where $c=1,2,4$ or 8 depending on the size of the matter multiplet of $\mathscr{N}$-extended supersymmetry. The resulting groups are summarised in table 2. 


\begin{tabular}{||c||c|c||c||}
\hline $\mathscr{N}$ & $G$ & $H_{\mathrm{R}}$ & $\operatorname{dim} \mathbb{R}^{c N}$ \\
\hline \hline 8 & $\mathrm{SO}(N)$ & $\mathrm{SO}(8)$ & $8 N$ \\
\hline 6 & $\mathrm{U}(N)$ & $\mathrm{SU}(4)$ & $8 N$ \\
\hline 5 & $\mathrm{Sp}(N)$ & $\mathrm{Sp}(2)$ & $8 N$ \\
\hline 4 & $\mathrm{Sp}(N) \times \mathrm{Sp}\left(\mathrm{N}^{\prime}\right)$ & $\mathrm{Sp}(1) \times \mathrm{Sp}(1)$ & $4 N+4 N^{\prime}$ \\
\hline 3 & $\mathrm{Sp}(\mathrm{N})$ & $\mathrm{Sp}(1)$ & $4 N$ \\
\hline 2 & $\mathrm{SU}(N)$ & $\mathrm{U}(1)$ & $2 N$ \\
\hline 1 & $\mathrm{SO}(N)$ & 1 & $N$ \\
\hline
\end{tabular}

Table 2: The global symmety and R-symmetry groups $G$ and $H_{\mathrm{R}}$ of three-dimensional $\mathscr{N}$ extended field theory and the dimension of the flat scalar manifolds.

Our notation is as follows: the fundamental representation of $\hat{G}$ splits up according to $A=(I, a)$, where $I$ is the fundamental representation of the R-symmetry group $H_{\mathrm{R}}$ and $a$ of the global symmetry group $G$. The scalar fields correspond to the non-compact generators and are denoted by $X^{I a}$. As these correspond to nilpotent generators, the associated scalar manifolds are flat in all cases we consider and the group $G$ acts as their global symmetry. For $\mathscr{N}=4$ one gets two copies of flat manifolds, composed of the two different types of matter multiplets.

In addition to ungauged theories with global supersymmetry, one can also obtain their gauged counterparts from supergravity. As shown in [24], one can derive $\mathscr{N}=8$ conformal as well as non-conformal gaugings and even massive deformations from the corresponding supergravity by taking the proper global limit. In the case of conformal gaugings, it can be seen that this requires the embedding tensor $\Theta$ to be a singlet of the R-symmetry group, i.e. to only take values in the symmetric product of the adjoint of the global symmetry groups $G$. Only the components $\Theta_{a b, c d}$ lead to a conformal gauging, while other components can lead to non-conformal gaugings and/or massive deformations. Indeed, in the analysis of [24] it was found that the other components of the embedding tensor had to be rescaled with the Newton's constant in order to avoid singular terms. This rescaling changes the mass dimension of these components, such that they had exactly the mass dimension of non-conformal gaugings and/or massive deformations. The components $\Theta_{a b, c d}$ that we will retain do not require such a rescaling and indeed correspond to conformal gaugings.

It remains to be seen which components $\Theta_{a b, c d}$ can be obtained from gauged supergravity. In [24] it was found for $\mathscr{N}=8$ that only the four-form representation in (2.6) gives rise to conformal gaugings. The other representations in (2.6) give rise to non-zero values for other components of the embedding tensor, involving the $R$-symmetry directions, and hence they spoil the conformal invariance. Therefore for $\mathscr{N}=8$ globally supersymmetric field theories we obtain conformal gaugings parametrised by an embedding tensor in the four-form representation of the global symmetry group $G$, which is $\mathrm{SO}(N)$ for the case of global supersymmetry. This is in precise agreement with the findings in the direct 
construction $[2-4,23]$.

A short analysis reveals that the situation is slightly different for the theories with less than $\mathscr{N}=8$ supersymmetry, in that one can use all representations of the supergravity embedding tensor to obtain conformal gaugings ${ }^{5}$. These are therefore classified by exactly the same representations that solve the linear constraint in the supergravity case, except that the linear constraint now refers to $G$ instead of $\hat{G}$. In more detail, we find the following conditions for the different cases:

- $\mathscr{N}=8$ field theory:

The embedding tensor takes values in the following representation of $G=\operatorname{SO}(N)$ :

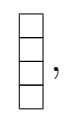

and as a consequence is totally anti-symmetric

$$
\Theta_{a b, c d}=\Theta_{[a b, c d]} .
$$

With the $\mathrm{SO}(N)$ structure constants

$$
\left.f^{a b, c d,}=-2 \delta^{[a}{ }_{[e} \delta^{b][c} \delta^{d]} f\right],
$$

the quadratic constraint (2.5) takes the explicit form

$$
\Theta_{a b, e}^{g} \Theta_{c d, g f}+\Theta_{a b, c}{ }^{g} \Theta_{e f, g d}-\Theta_{a b, f}^{g} \Theta_{c d, g e}-\Theta_{a b, d}{ }^{g} \Theta_{e f, g c}=0 .
$$

- $\mathscr{N}=6$ field theory:

The embedding tensor takes values in the following representations of $G=\mathrm{U}(N)$ :

$$
1 \oplus \square \bar{\square} \oplus \boxminus \bar{\square}
$$

and therefore is anti-symmetric in its two pairs of indices:

$$
\Theta_{a, c}^{b}{ }^{d}=\Theta_{[a, c]}^{b}{ }^{d} .
$$

With the $\mathrm{U}(N)$ structure constants

$$
f_{a}^{b},{ }_{c}^{d},{ }_{e}^{f}=i\left(\delta_{c}^{b} \delta_{a}{ }^{f} \delta_{e}{ }^{d}-\delta_{a}{ }^{d} \delta_{c}{ }^{f} \delta_{e}^{b}\right),
$$

the quadratic constraint (2.5) takes the explicit form

$$
\Theta_{c}{ }^{g}, e^{f} \Theta_{g}{ }^{d},{ }_{a}{ }^{b}-\Theta_{g}{ }^{d},{ }^{f}{ }^{f} \Theta_{c}{ }^{g},{ }^{b}{ }^{b}+\Theta_{a}{ }^{g}, e^{f} \Theta_{g}{ }^{b},{ }_{c}{ }^{d}-\Theta_{g}{ }^{b},{ }^{f} \Theta_{a}{ }^{g},{ }_{c}{ }^{d}=0 .
$$

- $\mathscr{N}=5$ field theory:

\footnotetext{
${ }^{5}$ The reason is that for lower $\mathscr{N}$, the corresponding components in the embedding tensor can be excited independently without inducing components in other blocks of the embedding tensor, see the appendix of [28] for the detailed decompositions. For $\mathscr{N}=8$ in contrast, a non-vanishing component $\square$ within $S O(N)$ induces components in the non-compact part of the embedding tensor which spoil the global limit.
} 
The embedding tensor takes values in the following representations of $G=\operatorname{Sp}(N)$ :

$$
1 \oplus \boxminus \oplus \boxminus,
$$

and hence satisfies the linear constraint

$$
\Theta_{(a b, c d)}=0 .
$$

With the $\operatorname{Sp}(N)$ structure constants

$$
f^{a b, c d,}{ }_{e f}=-2 \delta^{(a}{ }_{(e} \Omega^{b)(c} \delta^{d)}{ }_{f)},
$$

the quadratic constraint (2.5) takes the explicit form

$$
\Omega^{g h}\left(\Theta_{a b, e g} \Theta_{h f, c d}+\Theta_{a b, f g} \Theta_{h e, c d}+\Theta_{a b, c g} \Theta_{h d, e f}+\Theta_{a b, d g} \Theta_{h c, e f}\right)=0 .
$$

- $\mathscr{N}=4$ field theory:

As in the $\mathscr{N}=4$ supergravity case, the embedding tensor consists of three parts, $\Theta_{a b, c d}$, $\Theta_{a^{\prime} b^{\prime}, c^{\prime} d^{\prime}}$ and $\Theta_{a b, c^{\prime} d^{\prime}}$ that take values in the products of the adjoints of $\operatorname{Sp}(N)$ and $\operatorname{Sp}\left(N^{\prime}\right)$. The former two consist of the same representations (2.19) as in the $\mathscr{N}=5$ case, while the latter is unconstrained. The quadratic constraints can also be written in a form analogous to (2.22) using the $S p(N)$ and $S p\left(N^{\prime}\right)$ structure constants.

- $\mathscr{N}<3$ field theory:

As in the $\mathscr{N} \leq 3$ supergravities, the embedding tensor can take arbitrary values in the symmetric product of the adjoints of $G$. All consistent gaugings (satisfying the quadratic constraint) are compatible with supersymmetry.

In this way we have obtained a classification of the possible superconformal gaugings for different values of $\mathscr{N}$ in a uniform way, starting from the classification of the possible gaugings of supergravity. Of course, one still needs to solve the constraints for the embedding tensor. As we have discussed above, there are in general two approaches to solve these constaints, starting by either choosing for $\Theta$ the projector onto a subgroup, or by solving the linear constraint on the embedding tensor first. In both cases, one set of constraints is trivially satisfied while the other one becomes a rather non-trivial identity. Both approaches have been pursued in the literature and depending on the point of view, the remaining constraint (which is the linear one in the superpotential formalism of $[16,20]$ and the quadratic one in the 3-algebra formalism of $[3,5,21]$ ) has been referred to as fundamental identity, respectively.

It is interesting to compare our results to those obtained recently by the mechanism of supersymmetry enhancement. In this approach one starts from the superconformal theories with $\mathscr{N} \leq 3$ for which there is no restriction on the gauge group and the representation of the matter multiplets. In our context, this corresponds to the absence of a linear constraint on the embedding tensor. It was found that supersymmetry could be enhanced to $\mathscr{N}=4$ by certain restrictions on the gauge group and its representations [16]. These correspond to the linear constraint $\Theta_{(a b, c d)}=0$. Subsequently, it was noted that twisted hypermultiplets could be added and in fact are necessary for further 
supersymmetry enhancement $[18,30]$. The untwisted and twisted sector have to be taken identical to gain one further supersymmetry. In our notation, this corresponds to the identification of the three parts of the $\mathscr{N}=4$ embedding tensor leading to one $\mathscr{N}=5$ embedding tensor $\Theta_{a b, c d}$ subject to the same linear constraint (2.20). Yet further enhancement to $\mathscr{N}=6$ and $\mathscr{N}=8$ is possible by restricting to embedding tensors that satisfy the corresponding linear constraints (2.16) and (2.12), respectively.

The same superconformal gaugings can therefore be obtained in a methodical way from two independent and rather different approaches. In supergravity, the phenomenon of supersymmetry enhancement does not exist: one can not adjust the couplings of e.g. $\mathscr{N}=3$ supergravity to obtain an $\mathscr{N}=4$ theory. This can for example be seen from the different supergravity multiplets: the number of gravitini is different for these the-

ories. Nevertheless, the classification of supergravity gaugings reduces in the conformal limit to the same classification of superconformal gaugings that has been obtained from a global supersymmetry viewpoint. It is interesting to see that the analogous results have been obtained independently on the local and the global supersymmetry side. Using the conformal limit these two approaches can be related.

\section{Solving the constraints}

In this section we will show how to solve systematically the linear and quadratic constraints. We will first explain the general strategy and next discuss the cases for different values of $\mathscr{N}$ separately. Recently [20], a classification of the different superconformal theories has been given starting from $\mathscr{N}=4$ supersymmetry with special matter multiplets and making use of a relation with Lie superalgebras [16]. An alternative derivation for $\mathscr{N}=6$, of a more group-theoretical nature, can be found in [22]. Here we will use an approach directly based on the embedding tensor. Based on the relation with Lie superalgebras we will also uncover new $\mathscr{N}=4,5$ superconformal gaugings that correspond to exceptional cases.

Our starting point is an embedding tensor that has only directions in the global symmetry group $G$. The purpose of the embedding tensor is to project the Lie algebra generators of the global symmetry group onto the generators of the subgroup which is gauged. As explained in the previous section, this tensor must satisfy certain linear and quadratic constraints. Our strategy is to start from an embedding tensor which projects onto a given subgroup such that the quadratic constraint is automatically satisfied. In this case, the linear constraint becomes a non-trivial identity which decides if the gauging is a viable one.

For the classical Lie groups we will use the standard invariant tensors $\delta_{a b}=\delta_{b a}$ (orthogonal groups), $\delta_{a}{ }^{b}$ (unitary groups) and $\Omega_{a b}=-\Omega_{b a}$ (symplectic groups). Here $\delta$ denotes the Kronecker delta and $\Omega$ the anti-symmetric symplectic tensor with inverse tensor $\Omega^{a b}$, i.e. $\Omega_{a c} \Omega^{b c}=\delta_{a}{ }^{b}$. Besides these tensors we will also use special invariant tensors in the case of $\mathrm{SO}(7)$ and $G_{2}$ which will lead to the new $\mathscr{N}=4,5$ superconformal theories. Our first task is to construct, using the invariant tensors, the operators that project the Lie algebra generators of the global symmetry group onto the generators of the subgroup which is gauged. Furthermore we will also need the operators that project onto the singlet representation. These operators will be the building blocks from which 
we will construct the embedding tensor. In the case of the classical orthogonal, unitary and symplectic groups these building blocks are given by:

$$
\begin{array}{lll}
\mathrm{SO}(N) \text { singlet: } & \delta_{a b} \delta_{c d}, & \mathrm{SO}(N) \text { adjoint: } \delta_{c[a} \delta_{b] d}, \\
\mathrm{SU}(N) \text { singlet: } & \delta_{a}{ }^{b} \delta_{c}{ }^{d}, & \mathrm{SU}(N) \text { adjoint: }\left(\delta_{c}{ }^{b} \delta_{a}{ }^{d}-\frac{1}{N} \delta_{a}{ }^{b} \delta_{c}{ }^{d}\right), \\
\mathrm{Sp}(N) \text { singlet: } & \Omega_{a b} \Omega_{c d}, & \mathrm{Sp}(N) \text { adjoint: } \Omega_{c(a} \Omega_{b) d},
\end{array}
$$

For $\mathrm{SO}(4)$ there is an additional operator that projects onto the adjoint representation given by

$$
\mathrm{SO}(4) \text { adjoint: } \epsilon_{a b c d} \text {. }
$$

This operator will be needed in the construction of the $\mathscr{N}=8$ and one of the exceptional $\mathscr{N}=4,5$ superconformal theories.

Typically we will need to split the index $a$ according to a pair of indices $(i, \bar{i})$ :

$$
a \rightarrow(i, \bar{i}) \quad \text { with } \quad i=1, \ldots, m ; \bar{i}=1, \ldots, n
$$

corresponding to a bi-fundamental representation. These cases will be referred to as matrix models. Clearly, $n=1$ is a special case for which the matrix reduces to a vector, and the indices $a$ and $i$ coincide. In principle one could consider the index $a$ to represent a sum of an arbitrary set of representations of the gauge group other than those described above, but in accordance with the Lie superalgebra approach of Gaiotto and Witten [16] we do not find such solutions.

Having satisfied the quadratic constraint by employing the above building blocks, we now discuss the solution of the linear constraint for the different cases with decreasing number of supersymmetries separately. From the structure of globally supersymmetric theories (in contrast to supergravity), it is clear that theories with $\mathscr{N}$ supercharges can be seen as particular examples of theories with lower $\mathscr{N}$. For this reason we will not repeat the higher $\mathscr{N}$ examples when discussing the lower $\mathscr{N}$ theories.

- $\mathscr{N}=8$ superconformal gaugings:

In this case the embedding tensor contains only one irreducible component under $\mathrm{SO}(N)$, that is the 4-index anti-symmetric tensor $\Theta_{a b, c d}=\Theta_{[a b, c d]}$. Therefore, one cannot use the Kronecker delta $\delta_{a b}$ within $\Theta$.

One possibility is to make use of the special operator given in (3.2) and write

$$
\Theta_{a b, c d}=g \epsilon_{a b c d},
$$

for arbitrary coupling constant $g$. This restricts to $N=4$ and $\mathrm{SO}(4)$ gauging.

Another possibility is to consider a symplectic gauging and to construct an invariant embedding tensor of the form $\Theta_{a b c d} \sim \Omega_{[a b} \Omega_{c d]}$. However, according to eq. (3.1) this is not an $\operatorname{Sp}(N)$ projection operator. Therefore, the quadratic constraint will not be satisfied and one cannot consider this possibility. We conclude that for $\mathscr{N}=8$ one can only gauge $S O(4)$ or multiple copies thereof.

- $\mathscr{N}=6$ superconformal gaugings: 
In this case we are dealing with an embedding tensor $\Theta_{a}{ }^{b}{ }_{c}{ }^{d}$ that satisfies the linear constraint (2.16). Since the embedding tensor has both upper and lower indices we can use the invariant Kronecker delta $\delta_{a}{ }^{b}$ to build expressions for $\Theta$. This does not restrict to particular values of $N$. That is the basic reason why for $\mathscr{N}=6$ one can obtain gaugings for arbitrary $N[24]$.

The easiest way to find a solution that satisfies the linear constraint is to take

$$
\Theta_{a, c}^{b}{ }^{d}=g \delta_{[a}{ }^{[d} \delta_{c]}{ }^{b]}=\frac{g}{2}\left(\delta_{c}{ }^{b} \delta_{a}{ }^{d}-\frac{1}{N} \delta_{a}{ }^{b} \delta_{c}{ }^{d}\right)-\frac{(N-1)}{N} \frac{g}{2} \delta_{a}{ }^{b} \delta_{c}{ }^{d},
$$

for arbitrary coupling constant $g$. Note that the singlet operator becomes a U(1) projection operator. For $N>1$ this picks out all generators of $\mathrm{U}(N)$ and leads to a gauging of the full $\mathrm{U}(N)$ group. Note that, in order to satisfy the linear constraint (2.16), we must take a specific combination of the $\mathrm{SU}(N)$ and $\mathrm{U}(1)$ operators. By taking multiple copies thereof one obtains vector models with $\mathrm{U}\left(m_{1}\right) \times \mathrm{U}\left(m_{2}\right) \times \cdots$ gauging, where $m_{1}+m_{2}+\ldots=N$.

We next consider a matrix model describing the embedding $\mathrm{U}(m) \times \mathrm{U}(n) \subset \mathrm{U}(N=$ $m n)$ such that the scalars transform in the bi-fundamental representation $(m, n)$. We first try an embedding tensor that contains products of adjoints with singlets. However, one finds that one can not satisfy the linear constraint (2.16) with this Ansatz. For this we need to add a common U(1) factor that acts on both factors. We thus obtain

$$
\begin{aligned}
& \Theta_{(i, \bar{i})}^{(k, \bar{k})},{ }_{(j, \bar{j})}{ }^{(l, \bar{l})}=g \delta_{\bar{i}}^{\bar{k}} \delta_{\bar{j}}^{\bar{l}}\left(\delta_{i}{ }^{l} \delta_{j}{ }^{k}-\frac{1}{m} \delta_{i}{ }^{k} \delta_{j}{ }^{l}\right)-g \delta_{j}{ }^{l} \delta_{i}{ }^{k}\left(\delta_{j}^{\bar{k}} \delta_{\bar{i}}^{\bar{l}}-\frac{1}{n} \delta_{\bar{i}}^{\bar{k}} \delta_{j}^{\bar{l}}\right) \\
& -\frac{(m-n)}{m n} g \delta_{i}{ }^{k} \delta_{j}{ }^{l} \delta_{\bar{i}}^{\bar{k}^{k}} \delta_{\bar{j}}^{\bar{l}}
\end{aligned}
$$

for arbitrary coupling constant $g$. We deduce that the unitary matrix model desribes a $\mathrm{SU}(m) \times \mathrm{SU}(n) \times \mathrm{U}(1)$ gauging, corresponding to the $\mathrm{U}(m \mid n)$ model of [20]. For $m=n$, in which case the $\mathrm{U}(1)$ factor vanishes [22], this is the ABJM model of [19] .

Finally, we consider symplectic gaugings. Note that we can now raise and lower indices using the symplectic tensor. We first try an embedding tensor that only contains the adjoint of $\operatorname{Sp}(n)$. However, this does not satisfy the linear constraint (2.16) and we must add an additional $\mathrm{U}(1)$ factor:

$$
\Theta_{a b, c d}=g \Omega_{a b} \Omega_{c d}-g\left(\Omega_{c a} \Omega_{b d}+\Omega_{c b} \Omega_{a d}\right),
$$

where the first term on the right-hand-side corresponds to the U(1) gauging and where the term between round brackets corresponds to the $\operatorname{Sp}(n)$ gauging. This is precisely the so-called $\operatorname{OSp}(2 \mid n)$ model of $[20]$.

- $\mathscr{N}=5$ superconformal gaugings:

The global symmetry group for $\mathscr{N}=5$ is $\operatorname{Sp}(N)$. We first try to gauge the full $\operatorname{Sp}(N)$ using the Ansatz

$$
\Theta_{a b, c d}=g \Omega_{c(a} \Omega_{b) d},
$$

with arbitrary coupling constant $g$. This indeed solves the linear constraint (2.20) and leads to a vector model with $\operatorname{Sp}(N)$ gauging. Similarly, one can take multiple copies thereof with $\operatorname{Sp}\left(N_{1}\right) \times \operatorname{Sp}\left(N_{2}\right) \times \cdots$ gauging with $N_{1}+N_{2}+\ldots=N$. 
It turns out that the vector model is a special case of a matrix model with $\mathrm{SO}(m) \times$ $\operatorname{Sp}(n)$ gauging. The corresponding embedding tensor solving the linear constraint is given by

$$
\Theta_{(i \bar{i})(j \bar{j}),(k \bar{k})(l \bar{l})}=g\left(\delta_{k[i} \delta_{j] l} \Omega_{\overline{i j} \bar{j}} \Omega_{\bar{k} \bar{l}}+\delta_{i j} \delta_{k l} \Omega_{\bar{k}(\bar{i}} \Omega_{\bar{j}) \bar{l}}\right)
$$

for arbitrary coupling constant $g$. This is precisely the so-called $\operatorname{OSp}(m \mid n)$ model of [20]. Note that the relative strength between the $\mathrm{SO}(m)$ and $\mathrm{Sp}(n)$ terms is fixed by the linear constraint (2.20). Matrix models with $\mathrm{SO}(m) \times \mathrm{SO}(n)$ or $\mathrm{Sp}(m) \times \mathrm{Sp}(n)$ gauging cannot be constructed simply because one cannot embed these in the global symmetry group $\operatorname{Sp}(N)$.

A systematic way to construct solutions to the constraints on the embedding tensor comes from the link to Lie superalgebras observed in [16]. Namely, utilizing Lie superalgebras with (anti-)commutation rules

$$
\left\{Q_{a}, Q_{b}\right\}=\left(t^{\alpha}\right)_{a b} T_{\alpha}, \quad\left[T_{\alpha}, Q_{a}\right]=\eta_{\alpha \beta}\left(t^{\beta}\right)_{a}{ }^{b} Q_{b},
$$

where $\eta_{\alpha \beta}$ is the Cartan-Killing metric, the embedding tensor defined by

$$
\Theta_{a}^{b},{ }_{c}^{d}=\left(t^{\alpha}\right)_{a}^{b}\left(t^{\beta}\right)_{c}{ }^{d} \eta_{\alpha \beta}
$$

is guaranteed to satisfy the linear constraint as a consequence of the Jacobi identity $\left[\left\{Q_{a}, Q_{b}\right\}, Q_{c}\right]+$ perms $=0$. Applying this to the exceptional Lie superalgebras ${ }^{6}$ as presented in [37] in a convenient notation, we find the folllowing additional solutions to the constraints of the embedding tensor.

In the case of the Lie superalgebra $F(4)$, the embedding tensor reads (where $i, j, .$. refer to the spinor representation 8 of $\mathrm{SO}(7)$ and $\alpha, \beta, .$. denote an $\mathrm{SU}(2)$ doublet)

$$
\Theta_{i \alpha j \beta, k \gamma l \delta}=\frac{1}{12} \Gamma_{i j}^{m n} \Gamma_{k l}^{m n} \epsilon_{\alpha \beta} \epsilon_{\gamma \delta}+\delta_{i j} \delta_{k l} \epsilon_{\gamma(\alpha} \epsilon_{\beta) \delta}
$$

with $S O(7)$ Gamma-matrices $\Gamma^{m}$. This provides a solution to the linear constraint (2.20) and gives rise to a gauging of $\mathrm{SO}(7) \times \mathrm{SU}(2)$.

The second possibility corresponds to the Lie superalgebra $G(3)$. The embedding tensor is given by (where $i, j, .$. refer to the fundamental representation 7 of $G_{2}$ and $\alpha, \beta, .$. denote an $\mathrm{SU}(2)$ doublet)

$$
\Theta_{i \alpha j \beta, k \gamma l \delta}=\left(\delta_{i[k} \delta_{l] j}+\frac{1}{4} C_{i j k l}\right) \epsilon_{\alpha \beta} \epsilon_{\gamma \delta}+\delta_{i j} \delta_{k l} \epsilon_{\gamma(\alpha} \epsilon_{\beta) \delta}
$$

where $C_{i j k l}$ is the invariant tensor of ${ }^{7} G_{2}$. This leads to a $G_{2} \times \mathrm{SU}(2)$ gauge group ${ }^{8}$.

\footnotetext{
${ }^{6}$ The above results on solutions of the linear constraint make use of the classical Lie superalgebras $\mathrm{U}(m \mid n)$ (denoted by $\operatorname{spl}(m, n)$ in [37]) and $\operatorname{OSp}(m \mid n)$. There exist two other classes of Lie superalgebras, referred to as "strange superalgebras" in [38] and denoted by $P(n)$ and $Q(n)$. However, as the structure constants of these algebras do not fit the pattern exhibited in (3.10), the associated Jacobi identities do not correspond to the constraints on the embedding tensor. As such, these algebras do not give new solutions.

${ }^{7}$ In showing that the structure constants of $G(3)$, which can be found in [37], have the required form shown in (3.10), it is important to note that the Cartan-Killing form of $G_{2}$ involves the invariant tensor $C_{i j k l}$.

${ }^{8}$ Note that the gauging of a $G_{2} \subset \mathrm{SO}(7)$ subgroup in the $\mathscr{N}=8$ case is not possible because, although $C_{i j k l}$ is totally anti-symmetric and hence satisfies the linear constraint (2.12), the combination $\delta_{i[k} \delta_{l] j}+\frac{1}{4} C_{i j k l}$, which is needed for closure, does not satisfy the $\mathscr{N}=8$ linear constraint [23].
} 
Finally, the Lie superalgebra $D(2 \mid 1 ; \alpha)$ (referred to as $\operatorname{OSp}(4 \mid 2 ; \alpha)$ in [37]) gives a deformation of the $\mathrm{SO}(4) \times \mathrm{Sp}(1)$ gauging with embedding tensor

$$
\Theta_{i \alpha j \beta, k \gamma l \delta}=\left(\delta_{i[k} \delta_{l] j}+\gamma / 2 \epsilon_{i j k l}\right) \epsilon_{\alpha \beta} \epsilon_{\gamma \delta}+\delta_{i j} \delta_{k l} \epsilon_{\gamma(\alpha} \epsilon_{\beta) \delta}
$$

with $i, j,=1, . ., 4$ of $S O(4)$ and $\alpha, \beta=1,2$ of $S U(2)$. This example corresponds to deformation of the gauging of $\mathrm{SO}(4) \times \mathrm{Sp}(1)$ in the $\mathrm{OSp}(4 \mid 1)$ model. In standard notation $D(2 \mid 1, \alpha), \alpha$ corresponds to the ratio $(1+\gamma) /(1-\gamma)$ of the two coupling constants of $S O(4)$.

- $\mathscr{N}=4$ superconformal gaugings:

A noteworthy feature of the case of four supersymmetries is that there is a direct product structure. Each factor has a R-symmetry $\operatorname{Sp}(1)$ and a global symmetry group $\operatorname{Sp}(N)$. To distinguish the first sector from the second, so-called "twisted" sector, we use $a$ indices for $\operatorname{Sp}(N)$ and $a^{\prime}$ for the twisted $\operatorname{Sp}\left(N^{\prime}\right)$. We already mentioned that there are three kind of embedding tensors: those with only $a$-indices, those with only twisted $a^{\prime}$-indices and mixed embedding tensors with $a$ and $a^{\prime}$ indices.

Restricting first to the untwisted sector, the set of possible models coincides with those described above for $\mathscr{N} \geq 5$. The reason is that the $\mathscr{N}=4$ linear constraint coincides with that of $\mathscr{N}=5$. Hence for every solution with $\mathscr{N} \geq 5$ there is a corresponding solution with $\mathscr{N}=4$. The two classes with $\mathrm{SO}(m) \times \mathrm{Sp}(n)$ and $\mathrm{SU}(m) \times \mathrm{SU}(n) \times \mathrm{U}(1)$ were first described by [16]. In addition to these two regular classes, the three exceptional cases that occurred in $\mathscr{N}=5$ also make their appearance in $\mathscr{N}=4$. The expressions for the embedding tensor are identical to their $\mathscr{N}=5$ counterparts.

The situation changes if we also include the twisted sector [18]. First of all, a relatively trivial possibility is to include this without coupling to the untwisted sector. This allows for additional gaugings parametrised by $\Theta_{a^{\prime} b^{\prime}, c^{\prime} d^{\prime}}$, which also has to be of one of the above forms. More interesting is the possibility to couple to two sectors, using the offdiagonal components $\Theta_{a b, c^{\prime} d^{\prime}}=\Theta_{c^{\prime} d^{\prime}, a b}$. It is impossible to excite this component for generic gaugings in the untwisted and twisted sector; it can easily be seen that there are no possible terms with the correct symmetry properties. Indeed, an identification has to be made between the gaugings in the two sectors, as we will now illustrate.

For concreteness we will specify to a $\mathrm{SO}(m) \times \mathrm{Sp}(n)$ and $\mathrm{SO}\left(m^{\prime}\right) \times \mathrm{Sp}\left(n^{\prime}\right)$ gauging in both sectors, respectively. Upon identification of the two orthogonal sectors, i.e. $\mathrm{SO}(m) \simeq$ $\mathrm{SO}\left(m^{\prime}\right)$, one needs to include an off-diagonal term (where $a=\{i, \bar{i}\}$ and $a^{\prime}=\left\{i^{\prime}, \bar{i}^{\prime}\right\}$ and $\left.i \simeq i^{\prime}\right)$

$$
\Theta_{a b, c^{\prime} d^{\prime}}=g \delta_{k^{\prime}[i} \delta_{j] l^{\prime}} \Omega_{\bar{i} \bar{j}} \Omega_{\bar{k} \bar{l}} .
$$

This corresponds to a gauge group $\mathrm{Sp}(n) \times \mathrm{SO}(m) \times \mathrm{Sp}\left(n^{\prime}\right)$, where the (twisted) hypermultiplets are in the bifundamental of the first (last) two factors. Similarly, upon identification of the two symplectic sectors, i.e. $\operatorname{Sp}(n) \simeq \operatorname{Sp}\left(n^{\prime}\right)$, the following off-diagonal terms has to be included (where $a=\{i, \bar{i}\}$ and $a^{\prime}=\left\{i^{\prime}, \bar{i}^{\prime}\right\}$ and $\bar{i} \simeq \bar{i}^{\prime}$ )

$$
\Theta_{a b, c^{\prime} d^{\prime}}=g \delta_{i j} \delta_{k^{\prime} l^{\prime}} \Omega_{\bar{k}^{\prime}(\bar{i}} \Omega_{\bar{j}) \overline{l^{\prime}}} .
$$

In this case the gauge group is $\mathrm{SO}(m) \times \mathrm{Sp}(n) \times \mathrm{SO}\left(m^{\prime}\right)$ where again the (twisted) hypermultiplets are in the bifundamental of the first (last) two factors. By subsequent 


\begin{tabular}{||c||c|c||c||}
\hline $\mathscr{N}$ & $\Theta$ & gauge group & Lie superalgebra \\
\hline \hline 4,8 & $(3.4)$ & $\mathrm{SU}(2) \times \mathrm{SU}(2)$ & $\mathrm{U}(2 \mid 2)$ \\
\hline \hline 4,6 & $(3.6)$ & $\mathrm{SU}(m) \times \mathrm{SU}(n) \times \mathrm{U}(1)$ & $\mathrm{U}(m \mid n)$ \\
\hline 4,6 & $(3.7)$ & $\mathrm{SO}(2) \times \mathrm{Sp}(n)$ & $\mathrm{OSp}(2 \mid n)$ \\
\hline \hline 4,5 & $(3.9)$ & $\mathrm{SO}(m) \times \mathrm{Sp}(n)$ & $\mathrm{OSp}(m \mid n)$ \\
\hline 4,5 & $(3.12)$ & $\mathrm{SO}(7) \times \mathrm{SU}(2)$ & $F(4)$ \\
\hline 4,5 & $(3.13)$ & $G_{2} \times \mathrm{SU}(2)$ & $G(3)$ \\
\hline 4,5 & $(3.14)$ & $\mathrm{SO}(4) \times \mathrm{Sp}(1)$ & $D(2 \mid 1 ; \alpha)$ \\
\hline
\end{tabular}

Table 3: The equation number of the embedding tensor and gauge group of different superconformal models for $4 \leq \mathscr{N} \leq 8$ and the associated Lie superalgebra. For $\mathscr{N}=4$ we only give the untwisted models; when including the twisted sector non-trivial couplings such as (3.15) or (3.16) can also be introduced. For $\mathscr{N} \leq 3$, there are no restrictions on the gauge group.

applications of this construction one can obtain an (in)finite $\mathrm{SO}\left(m_{1}\right) \times \mathrm{Sp}\left(n_{1}\right) \times \mathrm{SO}\left(m_{2}\right) \times$ $\operatorname{Sp}\left(n_{2}\right) \times \cdots$ gauge group [18].

A distinct possibility is to identify both gauge groups, i.e. $\mathrm{SO}(m) \simeq \mathrm{SO}\left(m^{\prime}\right)$ and $\operatorname{Sp}(n) \simeq \operatorname{Sp}\left(n^{\prime}\right)$. In this case one needs the off-diagonal component to consist of both terms discussed above:

$$
\Theta_{a b, c^{\prime} d^{\prime}}=g \delta_{k^{\prime}[i} \delta_{j] l^{\prime}} \Omega_{\bar{i} \bar{j}} \Omega_{\bar{k} \bar{l}}+g \delta_{i j} \delta_{k^{\prime} l^{\prime}} \Omega_{\bar{k}^{\prime}(\bar{i}} \Omega_{\bar{j}) \bar{l}^{\prime}}
$$

Note that this leads to $\Theta_{a b, c d}=\Theta_{a b, c^{\prime} d^{\prime}}=\Theta_{a^{\prime} b^{\prime}, c^{\prime} d^{\prime}}$. In this case the untwisted and twisted hypermultiplets naturally combine into $\mathscr{N}=5$ multiplets and one finds supersymmetry enhancement [20], in this case to the solution (3.9).

A similar story holds for the $\mathrm{SU}(m) \times \mathrm{SU}(n) \times \mathrm{U}(1)$ gauging, where one can also employ the twisted sector to obtain a chain of unitary gauge groups [18]. In addition, the identification of both unitary groups in the untwisted and twisted sector leads to $\mathscr{N}=6$ supersymmetry [20], as the embedding tensor automatically satisfies the corresponding linear constraint (2.16). Finally, one can construct couplings between the untwisted and twisted sector in the case that these are given by one of the three exceptional cases.

\section{- $\mathscr{N} \leq 3$ superconformal gaugings:}

From the gauged supergravity models with $\mathscr{N}=1,2$ or 3 supersymmetries it follows that these is no linear constraint. Therefore, there is no restriction on the gauge group and matter content. Furthermore, interacting superconformal field theories not based on a gauging, and hence without Chern-Simons terms, are known to exist for $\mathscr{N}=1$ $[33,34]$. It would be interesting to consider the link with the corresponding deformations in supergravity. 
This finishes our discussion of how the constraints are solved for different values of $\mathscr{N}$. For the convenience of the reader we have summarised the different possibilities in table 3.

\section{An example: the $\mathscr{N}=6$ superconformal theory}

In this section we will present more details on our construction for the specific case of $\mathscr{N}=6$.

The $\mathscr{N}=6$ superconformal gauge theory was first discussed in [19]. Its $\mathscr{N}=2$ superspace formulation was presented in [35]. The explicit form of the supersymmetry rules have first been given in $[20,36]$. The theory contains $4 N$ complex scalars $X_{a I}$, where $I, J, \ldots=(1, \ldots, 4)$ and $a, b, \ldots=(1, \ldots, N)$, together with their complex conjugates $\left(X_{a I}\right)^{*}=X^{a I}$. They transform in the $(4, N)$ fundamental representation of $\mathrm{SU}(4) \times$ $\mathrm{U}(N)$. The fermions are given by two-component Dirac spinors $\psi_{I}^{a}$ describing $4 N$ complex fermionic degrees of freedom.

The corresponding action can be obtained by taking the conformal limit of $\mathscr{N}=6$ supergravity. In this limit the scalar manifold reduces to a flat space, and thus in the ungauged case this results in the free Lagrangian [24]

$$
\mathscr{L}=-\frac{1}{2} \partial^{\mu} X^{a I} \partial_{\mu} X_{a I}+\frac{1}{2} \bar{\psi}_{a}^{I} \gamma^{\mu} \partial_{\mu} \psi_{I}^{a}
$$

which exhibits $\mathscr{N}=6$ superconformal symmetry.

The conformal limit in the gauged case then corresponds to a gauging of (4.1). Its action takes the general form

$$
\begin{aligned}
\mathscr{L}= & -\frac{1}{2} D^{\mu} X^{a I} D_{\mu} X_{a I}+\frac{1}{2} \bar{\psi}_{a}^{I} \gamma^{\mu} D_{\mu} \psi_{I}^{a}+\mathscr{L}_{Y} \\
& -\frac{1}{2} \varepsilon^{\mu \nu \rho} A_{\mu}{ }^{\alpha} \Theta_{\alpha \beta}\left(\partial_{\nu} A_{\rho}{ }^{\beta}-\frac{1}{3} \Theta_{\gamma \delta} f^{\beta \delta}{ }_{\epsilon} A_{\nu}{ }^{\gamma} A_{\rho}{ }^{\epsilon}\right)-\frac{1}{3} A_{2}{ }^{a}{ }_{I}{ }^{J K} A_{2}{ }^{I}{ }^{I} J K
\end{aligned}
$$

where $A_{2}{ }^{a}{ }_{I}^{J K}=\left(A_{2 a}{ }^{I} J K\right)^{*}$, which introduces gauge vectors entering covariant derivatives and a Chern-Simons term. Besides, it requires Yukawa-couplings and a scalar potential, which takes a quadratic form in terms of a tensor $A_{2}$, which also enters the supersymmetry variation of the fermions. The covariant derivatives of the scalars are defined as follows

$$
\begin{aligned}
& D_{\mu} X_{a I}=\partial_{\mu} X_{a I}-\Theta_{a}{ }^{b}{ }_{, c}{ }^{d} A_{\mu d}{ }^{c} X_{b I}, \\
& D_{\mu} X^{a I}=\partial_{\mu} X^{a I}+\Theta_{b}{ }^{a},{ }_{,}{ }^{c} A_{\mu c}{ }^{d} X^{b I},
\end{aligned}
$$

and a similar definition applies to the covariant derivatives of the spinors. Here, we have used that the $\mathrm{U}(N)$ gauge fields are anti-hermitian, $\left(A_{\mu a}{ }^{b}\right)^{*}=-A_{\mu b}{ }^{a}$. The Yukawa couplings read explicitly

$$
\begin{aligned}
& \mathscr{L}_{Y}=-\frac{1}{2} \Theta_{a}{ }^{b},{ }^{d}{ }^{d} X_{d I} X^{c J} \bar{\psi}_{b}^{I} \psi_{J}^{a}+\frac{1}{4} \Theta_{a}{ }^{b},{ }^{d}{ }^{d} X_{d}{ }^{c} \bar{\psi}_{b}^{I} \psi_{I}^{a} \\
& +\frac{1}{8}\left(\epsilon_{I J K L} \Theta_{c}{ }^{a},{ }^{b}{ }^{b} X^{I c} X^{J d} \tilde{\psi}_{a}^{K} \psi_{b}^{L}+\text { h.c. }\right),
\end{aligned}
$$

where $\psi_{b}^{L}=\left(\psi_{L}^{b}\right)^{\star}$ and $\tilde{\psi}_{a}^{K}$ is defined in the appendix. Note that conformal invariance forbids the occurrence of quartic fermion terms. Finally, the tensor ${ }^{9} A_{2}{ }^{a}{ }_{I}{ }^{J K}$ defining the

\footnotetext{
${ }^{9}$ The notation $A_{2}$ is inherited from $D=3$ supergravity from which it arises in the conformal limit.
} 
scalar potential is given by

$$
A_{2}{ }^{a}{ }_{I}^{J K}(X)=-\frac{1}{2} \Theta_{b}{ }^{a},{ }_{c}{ }^{d}\left(X^{b J} X^{c K} X_{d I}+\delta_{I}{ }^{[J} X^{K] b} X_{d}{ }^{c}\right)
$$

where we have used the abbreviation $X_{a}{ }^{b} \equiv X_{a I} X^{b I}$.

The supersymmetry transformations leaving invariant (4.2) are given by

$$
\begin{aligned}
\delta X^{a I} & =\bar{\epsilon}^{I J} \psi_{J}^{a}, \\
\delta \psi_{I}^{a} & =\gamma^{\mu} D_{\mu} X^{a J} \epsilon_{I J}+A_{2}{ }^{a}{ }^{J K}(X) \epsilon_{J K}, \\
\delta A_{\mu a}{ }^{b} & =\frac{1}{2} \bar{\psi}_{a}^{I} \gamma_{\mu} X^{b J} \epsilon_{I J}-\text { h.c. }
\end{aligned}
$$

where the supersymmetry parameter $\epsilon_{I J}$ is in the real antisymmetric representation of $\mathrm{SU}(4)$ and satisfies a reality condition, see (A.4). They leave (4.2) invariant, provided the embedding tensor satisfies the linear constraint (2.16) and the quadratic constraints (2.18). Note that the action takes a 'universal' form in terms of the embedding tensor in that any particular gauging corresponds to a specific choice of $\Theta$ in (4.2), subject to the linear and quadratic constraints (2.16) and (2.18), respectively.

It is instructive to verify the supersymmetry of the action corresponding to (4.2). The lowest-order supersymmetry variation of the kinetic terms no longer vanishes due to the non-commutativity of the covariant derivatives. Up to a total derivative we obtain

$$
\delta \mathscr{L}_{\text {kin }}={ }_{\frac{1}{4}} \Theta_{b, d}{ }^{c}{ }^{c} \bar{\psi}_{a}^{I} \gamma^{\mu \nu} F_{\mu \nu c}{ }^{d} X^{b J} \epsilon_{I J}
$$

These variations are canceled by the supersymmetry variation of the gauge vectors in the Chern-Simons term. The variation of the gauge vectors inside the covariant derivatives gives rise to additional contributions linear in $\Theta$. These are canceled by taking the $\Theta-$ dependent terms (parameterized by $A_{2}$ ) of $\delta \psi_{I}^{a}$ in the variation of the fermion kinetic term and by taking the $\Theta$-independent term in the variation of the fermions in the Yukawa terms. This cancelation takes place provided the linear constraint (2.16) on $\Theta$ holds

We next consider the variations quadratic in $\Theta$. The variation of the Yukawa couplings leads to two types of terms, $\bar{\psi}_{a}^{I} \epsilon_{J K}$ and $\bar{\psi}_{a}^{I} \epsilon_{I J}$, i.e., with uncontracted or contracted SU(4) indices. The former terms vanish among themselves, which can be proven upon using linear combinations of the quadratic constraints (2.18) with different index permutations. Similarly, the latter terms cancel against the variations of the scalar potential.

Let us finally note that the supersymmetric action for all $\mathscr{N} \leq 5$ take the same universal form as (4.2), in which the Yukawa couplings and scalar potential are parameterized by the tensors $A_{2}$ and $A_{3}$ that can be obtained from the supergravity models of [28] as in [24].

\section{Discussion}

In this work we used the three-dimensional gauged supergravity models of $[27,28]$ to obtain information about superconformal gauge theories in three dimensions for an arbitrary number $\mathscr{N} \leq 8$ of supersymmetries. The embedding tensor characterizing the superconformal theory satisfies a linear and a quadratic constraint. For each solution 
of these constraints one obtains a consistent gauging. We solved the constraints using a simple tensor analysis and presented the gauge groups and matter content of the different superconformal theories. We find all the superconformal theories that occur in the recent classification of $[16,18,20,22]$. On top of that we find three new superconformal theories with $\mathscr{N}=4,5$ supersymmetry. These latter cases are suggested by the relation with the Lie superalgebras [16].

The supergravity approach allows to construct non-conformal gaugings and deformations as well [24]. These include (1) massive deformations of the superconformal theories and (2) standard Yang-Mills gauge theories. The massive deformations occur in two types: (1a) scalar massive deformations and (1b) vector massive deformation. In the former case one introduces mass parameters for a number of scalar fields. In the latter case one gauges translations corresponding to a number of scalar fields. This requires the introduction of new gauge vector fields, with a corresponding Chern-Simons term. In the gauge where the scalars are vanishing, the vector fields obtain a mass term in addition to their Chern-Simons term. By taking a non-conformal limit of gauged supergravity it can be shown that the scalar and vector mass parameters of the $\mathscr{N}=8$ superconformal theory occur in the following representations of the R-symmetry group $\mathrm{SO}(8)$ [24]:

$$
\text { scalar masses : } \quad \mathbf{3 5 _ { s }}, \quad \text { vector masses : } \mathbf{3} \mathbf{5}_{v} \text {. }
$$

Decomposing into $\mathrm{SU}(4) \times \mathrm{U}(1)$ and projecting onto $\mathrm{U}(1)$ singlets suggests that the $\mathscr{N}=6$ superconformal theory can be deformed by the following representations of the $\mathrm{SU}(4)$ R-symmetry group :

$$
\text { scalar masses : } \mathbf{1 5}, \quad \text { vector masses : } \mathbf{1 5} \text {. }
$$

Similarly, this leads one to expect the following representations of the $S p(2)$ R-symmetry group for $\mathscr{N}=5$ :

$$
\text { scalar masses : } \mathbf{5}, \quad \text { vector masses : } \mathbf{5} \text {. }
$$

The presence of a scalar mass term breaks the R-symmetry group to $S O(4) \times S O(\mathscr{N}-4)$ $[20,29-31]$. Continuing to lower $\mathscr{N}$ one could in this way classify massive deformations of all superconformal theories. It would be interesting to construct these deformations by taking the non-conformal limit of the gauged supergravity models of [28] and study their interplay with conformal and non-conformal gaugings.

\section{Acknowledgments}

We acknowledge discussions with Joaquim Gomis, Neil Lambert, Jaemo Park and Paul Townsend. E.A.B. thanks the physics department of Barcelona University for their kind support and hospitality. D.R. wishes to thank the Ecole Normale Superieure de Lyon for its hospitality. E.S. is grateful to University of Groningen, Ecole Normale Superieure de Lyon and SISSA for hospitality during the course of this work. This work was partially supported by the European Commission FP6 program MRTN-CT-2004-005104EU and by the INTAS Project 1000008-7928. In addition, the work of D.R. has been supported by MCYT FPA 2004-04582-C02-01 and CIRIT GC 2005SGR-00564. The work of H.S. was supported in part by the Agence Nationale de la Recherche (ANR). The research of E.S. is supported in part by NSF grant PHY-0555575. 


\section{A Notations and Conventions}

This appendix contains information about the notation and conventions pertaining to the $\mathscr{N}=6$ theory discussed in section 4 .

We choose the space-time metric to be $\eta=\operatorname{diag}(-++)$. The gamma matrices satisfy the Clifford algebra $\left\{\gamma^{\mu}, \gamma^{\nu}\right\}=2 \eta^{\mu \nu}$ and obey the identities

$$
\left(\gamma^{\mu}\right)^{\dagger}=\gamma_{0} \gamma^{\mu} \gamma_{0}, \quad\left(\gamma^{\mu}\right)^{T}=-C \gamma^{\mu} C^{-1}, \quad\left(\gamma^{\mu}\right)^{*}=B \gamma^{\mu} B^{-1}
$$

where $C^{T}=-C$ is the charge conjugation matrix and $B=-C \gamma_{0}$. Note that $C^{\dagger} C=$ $1, C^{*}=-C^{-1}$ and $B^{*} B=1$. In case of $U(N)$ symmetry, we use complex notation, i.e., the spinor fields are two-component Dirac spinors. A single Dirac spinor describes 2 real physical degrees of freedom. We define the Dirac conjugate as

$$
\bar{\psi}_{a}^{I}=\left(\psi_{I}^{a}\right)^{\dagger} i \gamma_{0}
$$

such that $\bar{\psi} \psi$ is a (real) Lorentz scalar. For Dirac spinors there is a second bilinear invariant, defined by

$$
\tilde{\psi}_{I}^{a} \psi_{J}^{b}=i\left(\psi^{T}\right)_{I}^{a} C \psi_{J}^{b} \quad \text { and } \quad \tilde{\psi}_{a}^{I} \psi_{b}^{J}=i\left(\psi^{T}\right)_{a}^{I} C^{-1} \psi_{b}^{J},
$$

where $\psi_{a}^{I}=\left(\psi_{I}^{a}\right)^{\star}$. In case of Majorana spinors, satisfying $\bar{\psi}=\psi^{T} C$, the two invariants defined in (A.2) and (A.3) coincide. The supersymmetry parameter satisfies a reality condition in order to be compatible with $\mathscr{N}=6$ supersymmetry,

$$
\left(\epsilon_{I J}\right)^{\star}=\epsilon^{I J}=\frac{1}{2} B \varepsilon^{I J K L} \epsilon_{K L} .
$$

Using this reality constraint, the supersymmetry transformation of the complex conjugate spinor $\psi_{a}^{I}$ for $\mathscr{N}=6$ reads

$$
\delta_{\epsilon} \psi_{a}^{I}=\frac{1}{2} B\left(\varepsilon^{I J K L} \gamma^{\mu} D_{\mu} X_{a J}+\varepsilon^{K L P Q} \bar{A}_{2 a}^{I} P Q\right) \epsilon_{K L}
$$

\section{References}

[1] J. H. Schwarz, "Superconformal Chern-Simons theories," JHEP 0411 (2004) 078 [arXiv:hep-th/0411077].

[2] J. Bagger and N. Lambert, "Modeling multiple M2's," Phys. Rev. D 75 (2007) 045020 [arXiv:hep-th/0611108].

[3] J. Bagger and N. Lambert, "Gauge Symmetry and Supersymmetry of Multiple M2Branes," Phys. Rev. D 77 (2008) 065008 [arXiv:0711.0955 [hep-th]].

[4] J. Bagger and N. Lambert, "Comments On Multiple M2-branes," JHEP 0802 (2008) 105 [arXiv:0712.3738 [hep-th]].

[5] A. Gustavsson, "Algebraic structures on parallel M2-branes," arXiv:0709.1260 [hepth]. 
[6] A. Gustavsson, "Selfdual strings and loop space Nahm equations," JHEP 0804 (2008) 083 [arXiv:0802.3456 [hep-th]].

[7] M. A. Bandres, A. E. Lipstein and J. H. Schwarz, "N = 8 Superconformal ChernSimons Theories," JHEP 0805 (2008) 025 [arXiv:0803.3242 [hep-th]].

[8] G. Papadopoulos, "M2-branes, 3-Lie Algebras and Plucker relations," JHEP 0805 (2008) 054 [arXiv:0804.2662 [hep-th]].

[9] J. P. Gauntlett and J. B. Gutowski, "Constraining Maximally Supersymmetric Membrane Actions," arXiv:0804.3078 [hep-th].

[10] B. M. Zupnik and D. G. Pak, "Superfield formulation of the simplest threedimensional gauge theories and conformal supergravities," Theor. Math. Phys. 77 (1988) 1070 [Teor. Mat. Fiz. 77 (1988) 97].

[11] E. A. Ivanov, "Chern-Simons matter systems with manifest $\mathrm{N}=2$ supersymmetry," Phys. Lett. B 268 (1991) 203.

[12] L. V. Avdeev, G. V. Grigorev and D. I. Kazakov, "Renormalizations in Abelian Chern-Simons field theories with matter," Nucl. Phys. B 382, 561 (1992); L. V. Avdeev, D. I. Kazakov and I. N. Kondrashuk, "Renormalizations in supersymmetric and non-supersymmetric non-Abelian Chern-Simons field theories with matter," Nucl. Phys. B 391, 333 (1993).

[13] D. Gaiotto and X. Yin, "Notes on superconformal Chern-Simons-matter theories," JHEP 0708 (2007) 056 [arXiv:0704.3740 [hep-th]].

[14] B. M. Zupnik and D. V. Khetselius, "Three-dimensional extended supersymmetry in harmonic superspace," Sov. J. Nucl. Phys. 47 (1988) 730 [Yad. Fiz. 47 (1988) 1147 .

[15] H. C. Kao, "Selfdual Yang-Mills Chern-Simons Higgs systems with an N=3 extended supersymmetry," Phys. Rev. D 50 (1994) 2881; H. c. Kao, "The Chern-Simons coefficient in supersymmetric non-Abelian Chern-Simons Higgs theories," Phys. Rev. D 60 (1999) 065013 [arXiv:hep-th/9904043].

[16] D. Gaiotto and E. Witten, "Janus Configurations, Chern-Simons Couplings, And The Theta-Angle in N=4 Super Yang-Mills Theory," arXiv:0804.2907 [hep-th].

[17] H. Fuji, S. Terashima and M. Yamazaki, "A New N=4 Membrane Action via Orbifold," arXiv:0805.1997 [hep-th].

[18] K. Hosomichi, K. M. Lee, S. Lee, S. Lee and J. Park, "N=4 Superconformal ChernSimons Theories with Hyper and Twisted Hyper arXiv:0805.3662 [hep-th].

[19] O. Aharony, O. Bergman, D. L. Jafferis and J. Maldacena, "N=6 superconformal Chern-Simons-matter theories, M2-branes and their gravity duals," arXiv:0806.1218 [hep-th].

[20] K. Hosomichi, K. M. Lee, S. Lee, S. Lee and J. Park, "N=5,6 Superconformal ChernSimons Theories and M2-branes on Orbifolds," arXiv:0806.4977 [hep-th]. 
[21] J. Bagger and N. Lambert, "Three-Algebras and N=6 Chern-Simons Gauge Theories," arXiv:0807.0163 [hep-th].

[22] M. Schnabl and Y. Tachikawa, "Classification of $\mathrm{N}=6$ superconformal theories of ABJM type," arXiv:0807.1102 [hep-th].

[23] E. A. Bergshoeff, M. de Roo and O. Hohm, "Multiple M2-branes and the Embedding Tensor," Class. Quant. Grav. 25 (2008) 142001 [arXiv:0804.2201 [hep-th]].

[24] E. A. Bergshoeff, M. de Roo, O. Hohm and D. Roest, "Multiple Membranes from Gauged Supergravity," arXiv:0806.2584 [hep-th].

[25] H. Nicolai and H. Samtleben, "Maximal gauged supergravity in three dimensions," Phys. Rev. Lett. 86 (2001) 1686 [arXiv:hep-th/0010076].

[26] H. Nicolai and H. Samtleben, "Compact and noncompact gauged maximal supergravities in three dimensions," JHEP 0104 (2001) 022 [arXiv:hep-th/0103032].

[27] H. Nicolai and H. Samtleben, " $\mathscr{N}=8$ matter coupled AdS(3) supergravities," Phys. Lett. B 514 (2001) 165 [arXiv:hep-th/0106153].

[28] B. de Wit, I. Herger and H. Samtleben, "Gauged locally supersymmetric D = 3 nonlinear sigma models," Nucl. Phys. B 671 (2003) 175 [arXiv:hep-th/0307006].

[29] J. Gomis, A. J. Salim and F. Passerini, "Matrix Theory of Type IIB Plane Wave from Membranes," arXiv:0804.2186 [hep-th].

[30] K. Hosomichi, K. M. Lee and S. Lee, "Mass-Deformed Bagger-Lambert Theory and its BPS Objects," arXiv:0804.2519 [hep-th].

[31] J. Gomis, D. Rodriguez-Gomez, M. Van Raamsdonk and H. Verlinde, "A Massive Study of M2-brane Proposals," arXiv:0807.1074 [hep-th].

[32] B. de Wit, A. K. Tollsten and H. Nicolai, "Locally supersymmetric D $=3$ nonlinear sigma models," Nucl. Phys. B 392 (1993) 3 [arXiv:hep-th/9208074].

[33] H. Nicolai, E. Sezgin and Y. Tanii, "Conformally Invariant Supersymmetric Field Theories On $\mathrm{S}^{P}$ x S ${ }^{1}$ And Super P-Branes," Nucl. Phys. B 305 (1988) 483.

[34] C. Fronsdal, “The Dirac Supermultiplet," Phys. Rev. D 26 (1982) 1988.

[35] M. Benna, I. Klebanov, T. Klose and M. Smedback, "Superconformal Chern-Simons Theories and $A d S_{4} / C F T_{3}$ Correspondence," arXiv:0806.1519 [hep-th].

[36] D. Gaiotto, S. Giombi and X. Yin, "Spin Chains in N=6 Superconformal ChernSimons-Matter Theory," arXiv:0806.4589 [hep-th].

[37] V. Rittenberg, "A Guide To Lie Superalgebras," Tubingen Colloq. (1977) 3

[38] L. Frappat, P. Sorba and A. Sciarrino, "Dictionary on Lie superalgebras," arXiv:hepth/9607161. 